\title{
Study on accumulation process of As atoms in InP/GaInAs/InP hetero-structures
}

\author{
Masao Tabuchi, Akiko Mori*, Hiroshi Tameoka*, Katsunori Fujii*, and Yoshikazu Takeda* \\ Venture Business Laboratory, Nagoya University, Furo-cho, Chikusa-ku, Nagoya 464-8603, Japan \\ Fax: 81-52-789-5430, e-mail: tabuchi@vbl.nagoya-u.ac.jp \\ *Graduate School of Engineering, Nagoya University, Furo-cho, Chikusa-ku, Nagoya 464-8603, Japan
}

The distributions of As atoms at InP/GaInAs interfaces were investigated by analyzing the X-ray CTR scattering spectra for the samples grown at different growth temperatures and with different $\mathrm{H}_{2}$-flushing times. From the results of the investigation, the adsorption and desorption processes of As atom on the GaInAs surface during the growth were discussed. Although the desorption of As from a surface progressed at all the investigated temperatures, 590, 620, and $650^{\circ} \mathrm{C}$, a part of the As atoms, about $1.2[\mathrm{ML}]$, remained on the surface even when the $\mathrm{H}_{2}$-flushing time was long. On the other hand, when the $\mathrm{H}_{2}$-flushing time was short, the amounts of As atoms were larger at higher growth temperatures, which suggested the decomposition of As precursor was enhanced at higher growth temperatures. All the results strongly indicated that the amount of As atoms incorporated in InP layer sensitively depends on the balance between the desorption and adsorption of As and that it is difficult to predict the degree of distribution of the As without a high resolution interface analysis like the X-ray CTR scattering measurement.

Key words: X-ray CTR scattering, buried interfaces of semiconductors, adsorption, desorption

\section{INTRODUCTION}

Hetero-structures of III-V compound semiconductors have been utilized widely for high performance devices such as HEMTs (high electron mobility transistors), lasers, and HBTs (heterojunction-bipolar transistors). For the high performance devices, highly defined hetero-structures, such as quantum well structures of which layer thicknesses are designed at a monolayer range, are epitaxially grown using advanced crystal growth technique. However, it is recognized that a compositional grading arises unexpectedly at the hetero-interfaces even when the hetero-structures are grown by OMVPE (organometallic vapor phase epitaxy) that is one of the most advanced semiconductor growth technique.

We have investigated the degree of the compositional grading at the hetero-interfaces using X-ray crystal truncation rod (CTR) scattering measurement. The X-ray CTR scattering measurement is a powerful technique to investigate the buried interfaces and determine the distributions of atoms quantitatively at an atomic-scale[1-7]. In these works, investigating $\mathrm{InP} / \mathrm{GaInAs}$ hetero-interfaces, we had shown that the compositional grading of group- $\mathrm{V}$ elements can be suppressed by controlling a $\mathrm{H}_{2}$-flushing time before the growth of InP on the GaInAs layer when the growth temperature was $620^{\circ} \mathrm{C}$.[8] The result suggested that As adsorption is an significant origin that causes the compositional grading at the $\mathrm{InP} / \mathrm{GaInAs}$ interface.

In this work, the adsorption of the As atoms on the GaInAs surface depending on the growth temperature is discussed via the study on the compositional grading at the upper InP/GaInAs interfaces in $\mathrm{InP} / \mathrm{Ga}_{0.47} \mathrm{In}_{0.53} \mathrm{As} / \mathrm{InP}$ structures using the X-ray CTR scattering method.

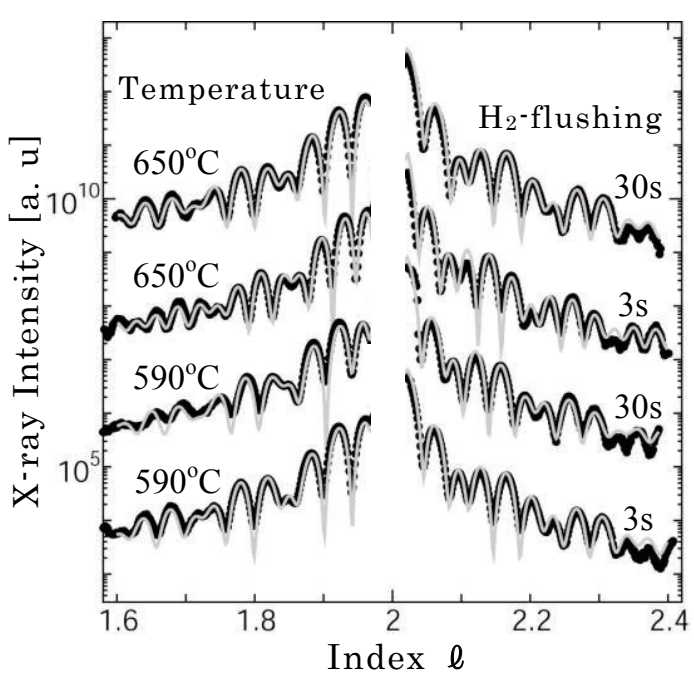

Fig. 1: Measured and theoretically calculated $\mathrm{X}$-ray CTR scattering spectra of the samples grown at 590 and $650^{\circ} \mathrm{C}$. 002 Bragg peaks of InP locate at $1=2.0$ although they were truncated in the figure.

\section{EXPERIMENTAL}

$\operatorname{InP}(50 \mathrm{ML}) / \mathrm{Ga}_{0.47} \mathrm{In}_{0.53} \mathrm{As}(15 \mathrm{ML}) / \mathrm{InP} \quad$ structure samples were grown on InP (001) just oriented substrates by OMVPE at 590,620 , and $650^{\circ} \mathrm{C}$. At the upper InP/GaInAs interface, $\mathrm{H}_{2}$-flushing time was changed from 3 to $30 \mathrm{sec}$, for which only $\mathrm{H}_{2}$ gas was flowed in order to flush out excess source gases from the OMVPE reactor. By analyzing the difference among the As distributions at the interfaces depending on the $\mathrm{H}_{2}$-flushing time, we can discuss the degree of As 


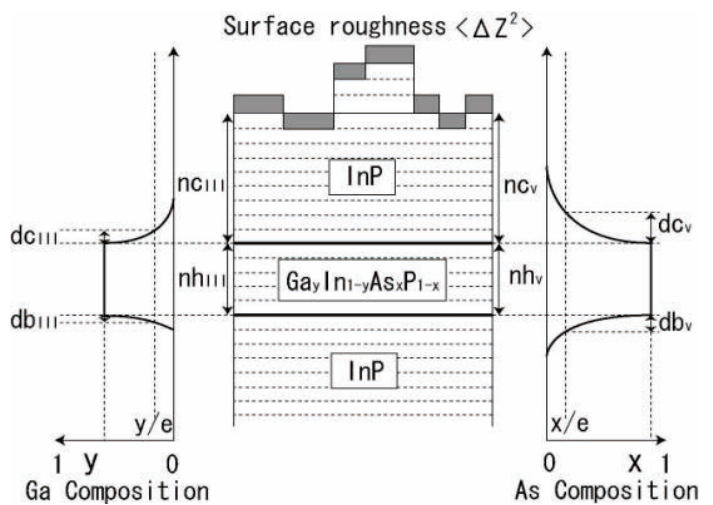

Fig. 2: A model structure to calculate a CTR scattering spectrum. The measured X-ray CTR scattering spectra were analyzed by comparing them with the theoretically calculated spectra based on this model. The model structure containes 10 parameters to describe the distributions of $\mathrm{Ga}$ and $\mathrm{As}$ in the $\mathrm{InP} / \mathrm{GaInAs} / \mathrm{InP}$ hetero-structure.

adsorption on the growing surface at a growth temperature.

TBAs, TBP, TMIn, and TEGa were used as source gases. Growth rate was $1.0 \mathrm{ML}$ (molecular layer)/s and $\mathrm{V} / \mathrm{III}$ ratio was fixed at 20 for all the samples.

The distributions of As atoms at the InP/GaInAs interfaces were analyzed from the results of the X-ray CTR scattering measurement. The X-ray CTR scattering measurement was carried out at the BL6A of the Photon Factory at the High Energy Accelerator Research Organization in Tsukuba, Japan. Wavelength of the X-ray was set at $0.1 \mathrm{~nm}$. A CCD camera was used to record the distribution of the scattered X-ray intensity around InP 002 Bragg peak.

In Figure 1, measured X-ray CTR scattering spectra of the $\mathrm{InP} / \mathrm{Ga}_{0.47} \mathrm{In}_{0.53} \mathrm{As} / \mathrm{InP}$ structure samples grown at $590^{\circ} \mathrm{C}$ and $650^{\circ} \mathrm{C}$ are shown as examples. The spectra

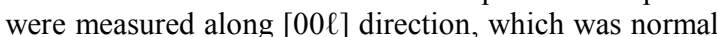
to the surface, in the reciprocal space. The peaks appeared at $\ell=2$ are 002 Bragg peak of InP, although they were not plotted near the Bragg point since the peaks were too high. As shown in Fig. 1, clear oscillations were observed in the range of $1.6<\ell<2.4$ for all the samples. The oscillation was caused by the interferences between reflected X-ray by InP and GaInAs layers. The difference of the amplitude of the oscillations reflected the degree of the distributions of atoms.

\section{RESULTS AND DISCUSSIONS}

The measured X-ray CTR scattering spectra were analyzed by comparing them with theoretically calculated spectra based on a model structure as shown in Fig. 2. Parameters that describe the distributions of atoms and roughness of the surface were contained in the model. The distributions of $\mathrm{Ga}$ and As atoms into the InP layers from GaInAs layer were assumed to be given by the simple form:

$$
x=x_{0} \exp \left(-\frac{d}{d_{0}}\right)
$$

where $x$ was Ga or As composition at $d$ [ML] away
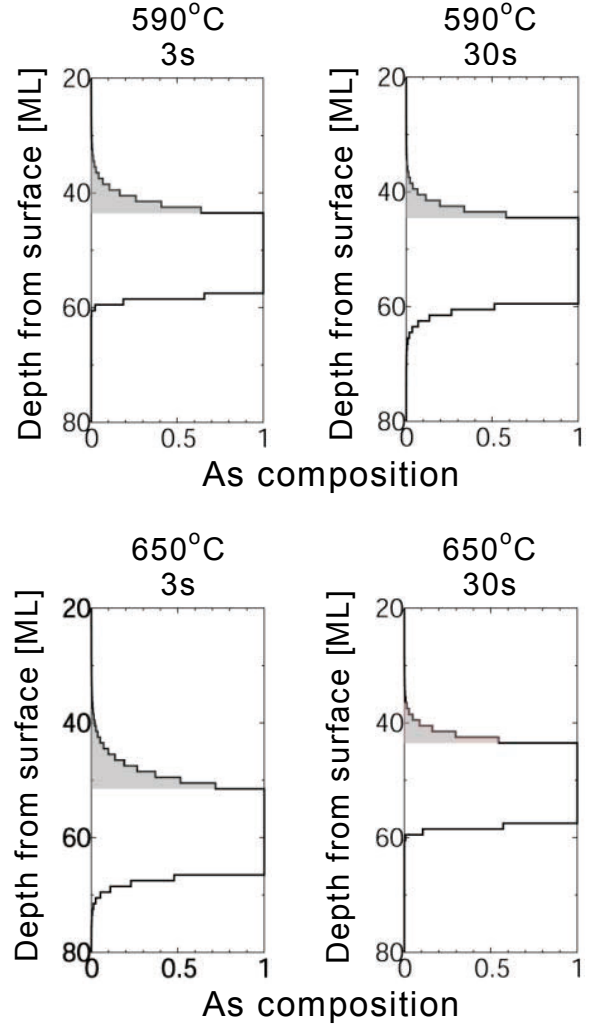

Fig.3: Composition profiles of As in the samples grown at 590 and $650^{\circ} \mathrm{C}$ obtained from the analysis of the X-ray CTR scattering spectra. The change of the degree of the compositional grading was small at $590^{\circ} \mathrm{C}$ with the change of the $\mathrm{H}_{2}$-flushing time. On the other hand, at higher growth temperature, $650^{\circ} \mathrm{C}$, the degree of compositional grading decreased when $\mathrm{H}_{2}$-flushing time was long. The amount of As in InP cap layer was calculated by summing up the As composition in the hatched region.

from an interface. $x_{0}$ was a $\mathrm{Ga}$ or As composition in the GaInAs layer, and $d_{0}$ was the parameter that represented the degree of the distribution. The theoretically calculated best-fitted spectra are also shown in Fig. 1 as grey lines.

Figure 3 shows examples of the distributions of As obtained from the analysis of the X-ray CTR scattering spectra. The hatched areas in Fig. 3, observed at the upper InP/GaInAs interface, represented the distributions of As in the InP cap layers. Amounts of the As incorporated in the InP cap layers were calculated and plotted in Fig. 4.

The distributions of As were also observed at the bottom GaInAs/InP interfaces. The amounts of As incorporated in the lower InP layers were from 0.7 to 1.2MLs. Since, the difference was within a standard error range and the change of the mount of As was not consistent between at 590 and $650^{\circ} \mathrm{C}$, it was difficult to discuss on the change of the lower interface structures. The distributions of As at lower interface were considered to be mainly caused by the exchange between $\mathrm{P}$ and As on top of InP before the growth of the GaInAs layer. Therefore, if a part of the distribution was caused by the roughness of the interface, the effect 


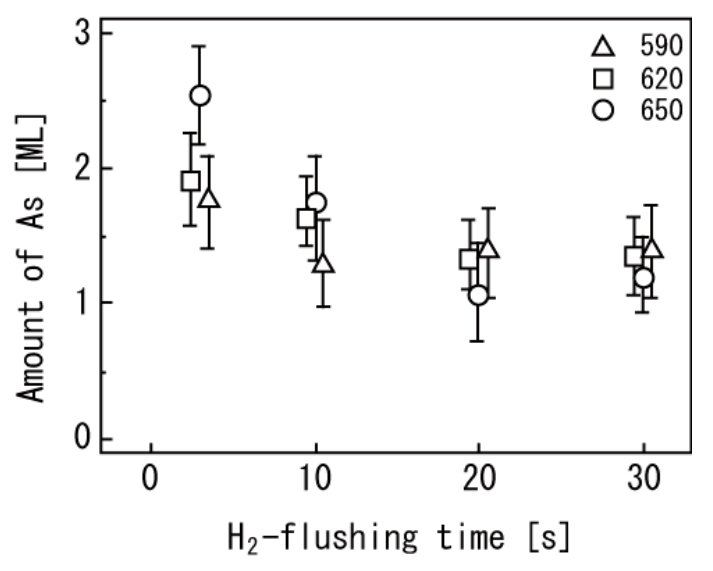

Fig.4: Relationships between the $\mathrm{H}_{2}$-flushing time and the amount of As incorporated in the InP cap layer. The amount of As decreased with the increase of the $\mathrm{H}_{2}$-flushing time at all the growth temperatures

was probably not so big on the amount of As incorporated in the InP layer at the upper interfaces.

Figure 4 shows the relationships between the amount of As incorporated in the InP cap layer and the $\mathrm{H}_{2}$-flushing time. The error bars were defined as regions where $\mathrm{R}$-factor increase by $20 \%$ at the border. As shown in Fig. 4, the amount of As decreased with the increase of the $\mathrm{H}_{2}$-flushing time at all the growth temperatures, i.e., 590, 620 , and $650^{\circ} \mathrm{C}$. It indicated that a part of As atoms adsorbing on the GaInAs surface desorbed during the $\mathrm{H}_{2}$-flushing.

When the $\mathrm{H}_{2}$-flushing time was long, the decrease of the amount of As was observed to saturate at about 1.2 ML. The result suggested that there should be other effects, such as, roughness of GaInAs surface and/or formation of stable surface reconstruction structure that determine minimal As concentration on a surface.

On the other hand, when the $\mathrm{H}_{2}$-flushing time was short, the amount of As was larger at higher growth temperatures. It is considered to be caused by the enhancement of the decomposition of As precursor at higher growth temperature resulting more adsorption of As[9].

All the results strongly indicated that the amount of the As atoms incorporated in the InP layer sensitively depends on the balance between the desorption and adsorption of As and that it is difficult to predict the degree of distribution of the As without a high resolution interface analysis like the X-ray CTR scattering measurement.

\section{CONCLUSIONS}

The distributions of As atoms at the $\mathrm{InP} / \mathrm{GaInAs}$ interfaces were investigated by analyzing the X-ray CTR scattering spectra for the samples grown at different growth temperatures and with different $\mathrm{H}_{2}$-flushing times. From the results of the investigation, the adsorption and desorption processes of As atoms on the GaInAs surface during the growth were discussed. Although the desorption of As from a surface progressed at all the investigated temperatures, 590,620 , and $650^{\circ} \mathrm{C}$, a part of the As atoms, about 1.2[ML] at all the temperatures, remained on the surface even when the $\mathrm{H}_{2}$-flushing time was long. On the other hand, when the $\mathrm{H}_{2}$-flushing time was short, the amount of As atoms were larger at higher growth temperature, which suggested the decomposition of As precursor was enhanced at higher growth temperatures. All the results strongly indicated that the amount of As atoms incorporated in InP layer sensitively depends on the balance between the desorption and adsorption of As and that it is difficult to predict the degree of distribution of the As without a high resolution interface analysis like the X-ray CTR scattering measurement.

\section{ACKNOWLEDGEMENTS}

This work was performed as a part of the projects 2007G557 and 2006G247 accepted by the Photon Factory Program Advisory Committee. This work was supported in part by the Grant-in-Aid for Scientific Research (S) \#18106001 and (B) \#19360006 from the Japan Society for the Promotion of Science.

\section{REFERENCES}

[1] I. K. Robinson, Rev. B, 33, 3830-3836(1986)

[2] I. K. Robinson, D. J. Tweet, Rep. Prog. Phys., 55, 599-651(1992).

[3] T. Shimura, and J. Harada, J. Appl. Cryst., 26, 151-158(1993).

[4] Y. Takeda, Y. Sakuraba, K. Fujibayashi, M. Tabuchi, T. Kumamoto, I. Takahashi, J. Harada, and H. Kamei, Appl. Phys. Lett., 66, 332-334(1995).

[5] M. Tabuchi, R. Takahashi, M. Araki, K. Hirayama, N. Futakuchi, Y. Shimogaki, Y. Nakano, and Y. Takeda, Appl. Surf. Sci., 159, 250-255(2000).

[6] M. Tabuchi, H. Kyouzu, M. Takemi, and Y. Takeda, Appl. Surf. Sci., 216, 526-531(2003).

[7] I. Yamakawa, R. Oga, Y. Fujiwara, Y. Takeda, and A. Nakamura, Appl. Phys. Lett., 84, 4436-4438(2004).

[8] A. Mori, Y. Ohtake, T. Ujihara, M. Tabuchi, and Y. Takeda, 19th. Inter. Conf. On Indium Phosphide and Ralated Materials (IPRM'07).

[9] M. Sugiyama, K. Kusunoki, Y. Shimogaki, S. Sudo, Y. Nakano, H. Nagamoto, K. Sugawara, K Tada, and H. Komiyama, Appl. Surf. Sci., 117/118, 746-752(1997).

(Received July 14, 2009;Accepted Septembe 18, 2009) 